\title{
Tracking of Mobile Devices through Bluetooth Contacts
}

\author{
Nikodin Ristanovic \\ EPFL, Switzerland
}

\author{
Dang-Khoa Tran \\ EPFL, Switzerland
}

\author{
Jean-Yves Le Boudec \\ EPFL, Switzerland
}

\begin{abstract}
We investigate if it is possible to reconstruct a mobile phone's mobility using its Bluetooth contacts with other mobile devices, some of which are equipped with GPS receivers. Our data mining analysis, based on two different data sets, shows that in certain environments coarse grained mobility of a signif cant portion of mobile phones can be obtained using this technique. For this reason, anyone capable of collecting Bluetooth and, if available, GPS logs for a large population of mobile phones, represents a serious privacy threat for all users with discoverable Bluetooth devices in the area.
\end{abstract}

\section{INTRODUCTION}

Low power consumption and security solutions built into Bluetooth led to the current situation where significant fraction of cell phones have their Bluetooth interfaces on all the time [1]. With the possible emergence of applications that involve mobile-to-mobile exchanges, the number of mobile phones with Bluetooth interfaces in discoverable mode could further increase. But, are we putting the little privacy that we have left at risk, by carelessly leaving our devices discoverable?

Mobile operators store customers' location records in CDRs. It is commonly believed that some phone manufacturers also collect location information. But, while we put a certain amount of trust in mobile operators, most of us would feel uncomfortable knowing that somebody unauthorized collects similar data. That would allow someone malicious to learn when we are outside our homes; advertising companies could get insight into our interests, etc. But how can anybody record my mobility, especially if I have a non-GPS phone?

Let's assume for a second that someone infected a

Permission to make digital or hard copies of all or part of this work for personal or classroom use is granted without fee provided that copies are not made or distributed for prof $t$ or commercial advantage and that copies bear this notice and the full citation on the f rst page. To copy otherwise, to republish, to post on servers or to redistribute to lists, requires prior specif c permission and/or a fee.

ACM CoNEXT Student Workshop, November 30, Philadelphia, USA. Copyright 2010 ACM 978-1-4503-0468-9/10/11 ...\$10.00. population of mobile phones with a piece of malicious software. Such software can force phones to log Bluetooth contacts with other phones [3]. Additionally, it can make GPS equipped phones log their locations. The recorded information can then be uploaded to a central location via data connection. A phone bot like this one could allow reconstruction of non-GPS phones' mobility (infected or not) as their locations can be estimated from the contacts with the GPS equipped phones.

We perform data mining analysis of two data sets. Our initial results show that even simple algorithms allow reconstruction of coarse mobility for a significant fraction of mobile devices present in the data sets.

\section{RELATED WORK}

Previously proposed approaches for wireless nodes localization exploit contacts with fixed reference points and knowledge of history, and their focus is location prediction. In [5] localization of nodes in 802.11 wireless networks is studied. The authors use information available at the link-layer of the 802.11 networks to train a Bayesian network and to calculate location. In $[4,6]$ the authors focus on wireless sensor networks.

In contrast, our focus is mobility reconstruction. We use no learning or prior knowledge and we obtain nodes' locations from contacts with mobile reference points.

\section{SYSTEM AND METRICS}

The existing malware for mobile phones is capable of stealing data and logging GPS coordinates or Bluetooth neighbors [2]. It propagates via Bluetooth, email, MMS, memory cards or infected applications. We assume that a certain population of mobile phones is infected by malicious software that forces the Bluetooth interface to stay visible and to periodically run the neighbor discovery mechanism. The recorded contacts are logged and uploaded to a central server via the phones' data connections. Some of the infected phones are equipped with GPS receivers; we refer to them as anchors. In addition to logging contacts, they log and upload their locations. Our goal is to determine if the opportunistically collected contacts are sufficient to reconstruct the 
mobility of the tracked devices, i.e. the infected nonGPS phones. We are interested in two measures:

- Mean localization error is the average distance between real and estimated locations of tracked devices.

- Mean inter-anchor time is the average interval between two consecutive contacts, which occur between a tracked device and any of the anchors.

\section{LOCALIZATION ALGORITHM}

We design an algorithm that does not require training and knowledge of contact history. The algorithm exploits the fact that a contact between a tracked device and an anchor roughly determines the location of the tracked device, as the location of the anchor is known and the Bluetooth range is $\sim 10 \mathrm{~m}$. Additionally, we assume that all the devices move at moderate speeds.

For these reasons, we consider the location of a tracked device $d_{1}$ to be known at time $t$ iff: (i) a direct contact between $d_{1}$ and an anchor is recorded within the time window $[t-\Delta t, t+\Delta t]$ or (ii) a contact between $d_{1}$ and a tracked device $d_{2}$ is recorded at time $t$, another contact between $d_{2}$ and an anchor is recorded at time $t_{2}$ and $t \in\left[t_{2}-\Delta t, t_{2}+\Delta t\right]$.

\section{USED DATA SETS AND RESULTS}

We evaluate our algorithm and assumptions using two data sets. The first one was collected by 20 nodes, during a three day hiking trip. The hikers were moving through a large region in disorderly fashion. Their devices periodically logged Bluetooth contacts and GPS coordinates. The second data set is a two-week contact trace collected at the university campus by 60 mobile devices (for 10 of them GPS records exist).

We present only a part of our results for the first data set. The availability of GPS records for all devices in this data set allows us to estimate the localization error.

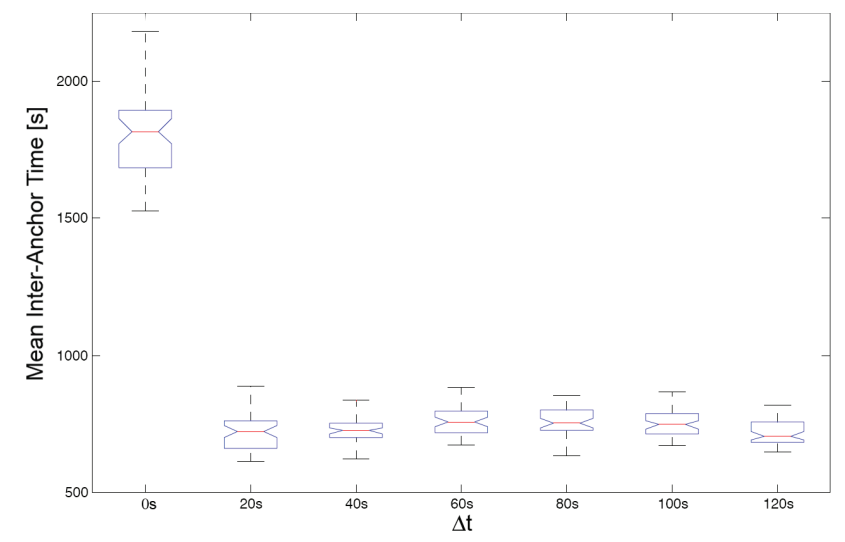

Figure 1: The average period during which the location of a tracked device is unknown.

When calculating the estimated locations of tracked devices we apply the following strategy: We select 5

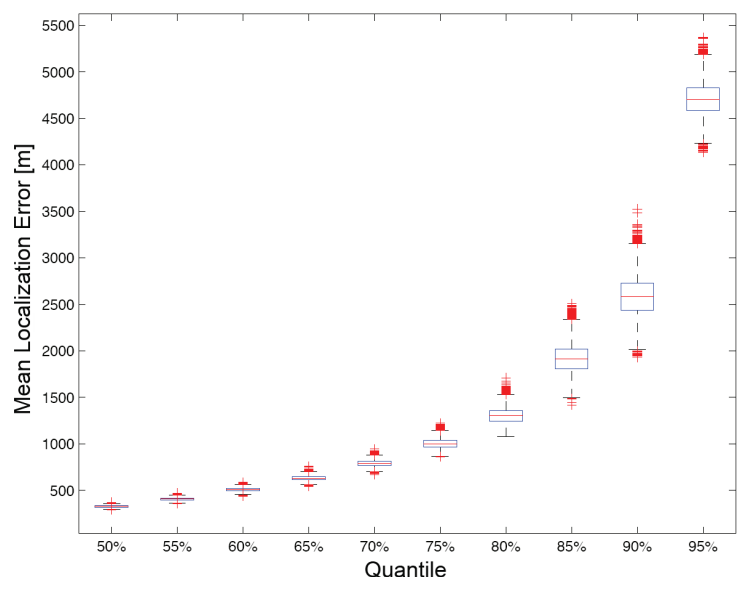

Figure 2: The localization error quantiles.

out of 20 devices to be the anchors whose positions are known. The positions of other 15 tracked devices are reconstructed from the contact files using our algorithm. Anchor's positions between the logged locations are inferred using interpolation. The estimated positions are then compared with the logged positions of tracked devices, in order to obtain the localization error. We run 50 simulations, each with 5 randomly selected anchors.

The average inter-anchor times for different values of parameter $\Delta t$ are shown in Figure 1. Increasing the value of $\Delta t$ from $0 s$ to $20 s$ allows for "indirect" contacts between tracked and anchor devices (as defined in Section 4) and significantly increases the number of location entries for the tracked devices. Further augmentation of $\Delta t$ brings little improvement.

Some tracked phones can be localized with higher precision than the others. For this reason, we calculate the localization error quantiles. Figure 2 contains the quantiles for $\Delta t=80 \mathrm{~s}$ (medians, 25th and 75th percentiles (box edges), extreme points (whiskers) and outliers (crosses)). We see that $50 \%$ of tracked devices can be localized within $300 \mathrm{~m}$ and $70 \%$ within $800 \mathrm{~m}$.

\section{REFERENCES}

[1] G. Ananthanarayanan and I. Stoica. Blue-fi: enhancing wi-fi performance using bluetooth signals. In Proc. MobiSys '09.

[2] Symbianpoint. http://symbianpoint.com/types-latestlist-mobile-viruses.html.

[3] BlueAttacks. http://gcn.com/articles/2005/07/20/amenu-of-bluetooth-attacks.aspx.

[4] S. Guha, R. Murty and E. Gun Sirer. Sextant: a unified node and event localization framework using non-convex constraints. In Proc. MobiHoc 05.

[5] A. Haeberlen, E. Flannery, A. M. Ladd, A. Rudys, D. S. Wallach and L. E. Kavraki. Practical robust localization over large-scale 802.11 wireless networks. In MobiCom 04 .

[6] G. Mao, B. Fidan, D. Towsley and B. D. O. Anderson. Wireless sensor network localization techniques. Computer Networks, 2007. 